\title{
FRANÇOIS DE THÉLIN
}

\section{Résultats d'existence et de non-existence pour la solution positive et bornée d'une e.d.p. elliptique non linéaire}

\author{
Annales de la faculté des sciences de Toulouse $5^{e}$ série, tome $8, \mathrm{n}^{\circ} 3$ \\ (1986-1987), p. 375-389 \\ <http://www.numdam.org/item?id=AFST_1986-1987_5_8_3_375_0>
}

(C) Université Paul Sabatier, 1986-1987, tous droits réservés.

L'accès aux archives de la revue «Annales de la faculté des sciences de Toulouse » (http://picard.ups-tlse.fr/ annales/) implique l'accord avec les conditions générales d'utilisation (http://www.numdam.org/conditions). Toute utilisation commerciale ou impression systématique est constitutive d'une infraction pénale. Toute copie ou impression de ce fichier doit contenir la présente mention de copyright.

\section{NumDam}

Article numérisé dans le cadre du programme

Numérisation de documents anciens mathématiques

http://www.numdam.org/ 


\title{
Résultats d'existence et de non-existence pour la solution positive et bornée d'une e.d.p. elliptique non linéaire
}

\author{
FranÇOIS DE ThELIN (1)
}

RÉSUME. - Dans cet article, nous obtenons quelques résultats d'existence et de non existence de la solution positive et bornée $u \in W_{0}^{1, p}(\Omega)$ de l'equation :

$$
\operatorname{div}\left(|\nabla u|^{p-2}\right)+g(x, u)=0 .
$$

Pour prouver les résultats d'existence, nous utilisons soit le théorème de Passe-Montagne, soit le construction explicite de sur et sous-solutions. Les résultats de non existence sont des conséquences d'une identité de type Pohozaev.

ABstract - - In this paper we obtain some existence and nonexistence results for bounded positive solutions $u \in W_{0}^{1, p}(\Omega)$ of the equation:

$$
\operatorname{div}\left(|\nabla u|^{p-2}\right)+g(x, u)=0 .
$$

Proving the existence results, either we use the Pass-Mountain lemma or we explicitly construct subsolutions and supersolutions of (E). The nonexistence result follows from a $\mathrm{POHOzAEV}$ identity.

\section{Introduction}

Soient $\Omega$ un ouvert borné régulier de $\mathbf{R}^{N}$ et $1<p<\infty$. Dans cet article, nous étudions l'existence ou la non-existence d'une solution $u$ positive et bornée, $u \in W_{0}^{1, p}(\Omega)$ de :

$$
\Delta_{p} u+g(x, u)=0 \quad \text { dans } \Omega
$$

où $F(\nabla u)=|\nabla u|^{p-2} \nabla u, \Delta_{p} u=\operatorname{div} F(\nabla u)$.

(1) Laboratoire d'Analyse Numérique, Université Paul Sabatier, 31062 Toulouse Cedex. 
Le cas $p=2$ a été étudié par de nombreux auteurs. Pour $p \neq 2$ et $g(x, u)=\lambda u^{\gamma-1}$, l'étude de l'existence de la solution a d'abord été entreprise par OtANi [10] en dimension 1, puis De Thélin [17] en dimension $N$; l'étude de la non-existence, basée sur une égalité de type PoHozaev [13] a été faite indépendamment par De ThÉLIN [17] et NI-SERRIN [7,8] pour des solutions radiales dans $\mathbf{R}^{N}$, puis complétée par OTANI [12] pour un ouvert quelconque.

Le but de ce papier est de présenter un ensemble de résultats d'existence et de non existence en dimension $N$, généralisant les précédents $[7,8,10$, 12] sans se limiter au seul cas $g(x, u)=\lambda u^{\gamma-1}$. On reprend plusieurs idées d'OTANI [12] et l'essentiel des énoncés de ses théorèmes.

Dans le cas particulier $g(x, u)=\lambda u^{\gamma-1}$, on retrouve les résultats connus :

- existence pour $\quad \gamma(N-p)<N p$

- non existence pour $\gamma(N-p) \geq N p$ lorsque $\Omega$ est un ouvert strictement étoilé.

Rappelons enfin qu'un théorème de DiAz-SAA [4] permet d'affirmer que la solution de notre problème est unique si $u \rightarrow \frac{g(x, u)}{u^{p-1}}$ est strictement décroissante.

Par ailleurs, pour l'étude du problème de la première valeur propre de $\Delta_{p}$, on peut voir [11] et [18].

\section{Existence}

Soient $\Omega$ un ouvert borné régulier de $\mathbf{R}^{N}, 1<p<+\infty$ et $g: \Omega \times \mathbf{R} \rightarrow \mathbf{R}$ est une fonction de Carathéodory.

Nous nous intéressons au problème suivant :

$$
(\mathcal{P})\left\{\begin{array}{l}
\text { Trouver } u \in W_{0}^{1, p}(\Omega), \quad u \geq 0, \quad u \not \equiv 0 \text { telle que : } \\
\Delta_{p} u+g(x, u)=0 \text { dans } \Omega .
\end{array}\right.
$$

THÉORÈME 1. - On suppose qu'il existe $\gamma_{1}$ et $\gamma_{2}: 1<\gamma_{1}<\gamma_{2}<p$, $\lambda_{1}>0, \lambda_{2}>0$ et $\mu \geq 0$ tels que :

(i) $\forall x \in \Omega, \forall u \geq 0: \lambda_{1} u^{\gamma_{1}-1} \leq g(x, u)$,

(ii) $\forall x \in \Omega, \forall u \geq 0: g(x, u) \leq \mu+\lambda_{2} u^{\gamma_{2}-1}$.

Alors $(\mathcal{P})$ admet au moins une solution $u$. De plus $u \in L^{\infty}(\Omega)$, ne s'annule pas dans $\Omega$, et possède les propriétés de régularité suivante :

Si $1<p \leq 2, u \in \mathcal{C}^{1, \alpha}(\Omega) \cap W_{\text {loc }}^{2, p}(\Omega)$;

$$
\text { si } p>2, \quad u \in \mathcal{C}^{1, \alpha}(\Omega) \cap B_{\infty, l o c}^{p^{*}, p}(\Omega) \text {. }
$$


Résultats d'existence et de non-existence pour une e.d.p. elliptique non linéaire

Si en outre la fonction : $u \rightarrow \frac{g(x, u)}{u^{\gamma_{2}-1}}$ est strictement décroissante, cette solution est unique.

Remarque. - Ici $p^{*}=\frac{p}{p-1}$ et $B_{\infty}^{p^{*}, p}$ est l'espace de Besov $\left[W^{1, p}, W^{2, p}\right]_{p^{*}-1, \infty}$ défini par interpolation.

Nous dirons que $\bar{u} \in W^{1, p}(\Omega)$ [resp. $\underline{u} \in W^{1, p}(\Omega)$ ] est sursolution de $\mathcal{P}$ $\left[\right.$ resp. sous solution de $\mathcal{P}$ ] si $\Delta_{p} \bar{u}+g(x, \bar{u}) \leq 0\left[\operatorname{resp} . \Delta_{p} \underline{u}+g(x, \underline{u}) \geq 0\right.$ ] dans $\Omega$ et si $\bar{u}>0$ sur $\partial \Omega$ [resp. $\underline{u} \leq 0$ sur $\partial \Omega$ ].

La démonstration du théorème 1 utilise les lemmes suivants.

LEMмE 1.- Sous l'hypothèse (ii), pour tout $M>0,(\mathcal{P})$ admet une sursolution $\bar{u}$ telle que :

$$
\forall x \in \Omega, \quad \bar{u}(x) \geq M
$$

Démonstration. - Soit $R>0$ tel que $\Omega \subset B(0, R)$ et cherchons $\bar{u}$ sous la forme : $\bar{u}(x)=\varphi(r)=a r^{p^{*}}+b$ avec $r=|x|, a<0, b>0$. On aura bien $\bar{u}(x) \geq M$ si on prend $a=-\frac{b-M}{R^{p^{*}}}$.

De manière générale, on $\mathrm{a}$ :

$$
\Delta_{p} \bar{u}+g(x, \bar{u})=(p-1)\left|\varphi^{\prime}(r)\right|^{p-2} \varphi^{\prime \prime}(r)+\frac{(N-1)}{r}\left|\varphi^{\prime}(r)\right|^{p-2} \varphi^{\prime}(r)+g(x, \bar{u}) .
$$

Il vient donc ici :

$$
\begin{aligned}
\Delta_{p} \bar{u}+g(x, \bar{u}) & \leq-N\left|a p^{*}\right|^{p-1}+\mu+\lambda_{2}\left(a r^{p^{*}}+b\right)^{\gamma_{2}-1} \\
& \leq-N\left(\frac{(b-M) p^{*}}{R^{p^{*}}}\right)^{p-1}+\mu+\lambda_{2} b^{\gamma_{2}-1} .
\end{aligned}
$$

Sachant que $p>\gamma_{2}$, on pourra choisir $b$ assez grand pour que

$$
\Delta_{p} \bar{u}+g(x, \bar{u}) \leq 0 . \square
$$

LEMмE 2.-Sous l'hypothèse (i), pour tout $M>0, \mathcal{P}$ admet une sous solution $\underline{u} \not \equiv 0$ telle que :

$$
\forall x \in \Omega, \quad 0 \leq \underline{u}(x) \leq M .
$$


Démonstration. - Supposons pour simplifier les notations que $0 \in \Omega$ et considérons $R>0$ tel que $B(0, R) \subset \Omega$. Cherchons $\underline{u}$ sous la forme :

$$
\begin{aligned}
& \underline{u}(x)= \begin{cases}\alpha|x|^{p^{*}}+\beta & \text { pour } 0 \leq|x| \leq \frac{N R}{N+1} \\
\delta(R-|x|)^{p^{*}} & \text { pour } \frac{N R}{N+1}<|x| \leq R \\
0 & \text { pour } x \in \Omega \backslash B(0, R)\end{cases} \\
& \text { avec }\left\{\begin{array}{l}
\beta=\left(\frac{\lambda_{1} R^{p}}{(N+1)^{\gamma_{1}}\left(p^{*}\right)^{p-1}}\right)^{1 /\left(p-\gamma_{1}\right)} \\
\alpha=-\frac{\beta}{R^{p^{*}}}\left(\frac{N+1}{N}\right)^{p^{*}-1} \\
\delta=-\alpha N^{p^{*}-1}
\end{array}\right.
\end{aligned}
$$

Les deux dernières relations assurent que $\underline{u} \in \mathcal{C}^{1}(\bar{\Omega})$; de plus $\underline{u} \geq 0, u \not \equiv 0$ dans $\Omega$ et $\underline{u}=0$ sur $\partial \Omega$.

Sachant que $p>\gamma_{1}$, on peut choisir $R$ assez petit pour que $\beta \leq M$ et donc $\underline{u} \leq M$ dans $\Omega$.

De plus, pour $\frac{N R}{N+1} \leq|x| \leq R$, il vient avec (1) :

$$
\begin{aligned}
\Delta_{p} \underline{u}+g(x, \underline{u}) & =\left(\delta p^{*}\right)^{p-1}\left(1-\frac{(N-1)(R-r)}{r}\right)+g(x, \bar{u}) \\
& \geq\left(\delta p^{*}\right)^{p-1}\left(N-\frac{(N-1)(N+1)}{N}\right) \geq 0 .
\end{aligned}
$$

Et pour $0 \leq|x| \leq \frac{N R}{N+1}$ :

$$
\begin{aligned}
\Delta_{p} \underline{u}+g(x, u) & \geq-N\left|\alpha p^{*}\right|^{p-1}+\lambda_{1}\left(\alpha\left(\frac{N R}{N+1}\right)^{p^{*}}+\beta\right)^{\gamma_{1}-1} \\
& \geq-\frac{N+1}{R^{p}}\left(\beta p^{*}\right)^{p-1}+\lambda_{1}\left(\frac{\beta}{N+1}\right)^{\gamma_{1}-1}=0
\end{aligned}
$$

par choix de $\beta$. D'où le résultat.

Démonstration du théorème 1 . - Il résulte des lemmes 1 et 2 qu'il existe $\bar{u}$ sursolution et $\underline{u}$ sous solutions de $(\mathcal{P})$ telles que $\underline{u} \leq \bar{u}$. D'après le théorème de Deuel et Hess [2], il existe $u \in W_{0}^{1, p}(\Omega)$ telle que $\underline{u} \leq u \leq \bar{u}$ et :

$$
\Delta_{p} u+g(x, u)=0 \text {. }
$$


Résultats d'existence et de non-existence pour une e.d.p. elliptique non linéaire

Evidemment $u \in L^{\infty}(\Omega)$; d'après TolksDorF [19], il existe alors $\alpha>0$ tel que $u \in \mathcal{C}^{1, \alpha}(\Omega)$. Il résulte donc du principe du maximum de VAZQuez [20] que :

$$
\forall x \in \Omega, \quad u(x)>0 .
$$

Par ailleurs pour $1<p \leq 2, u \in W_{\operatorname{loc}}^{2, p}(\Omega)[16]$ et pour $p>2, u \in$ $B_{\infty, \text { loc }}^{p^{*}, p}(\Omega)[14]$.

Enfin l'unicité résulte d'un théorème de Diaz et SAA [4].

THÉORÈME 2.-On suppose :

(i) $\forall x \in \Omega, \forall u>0, g(x, u)>0$;

(ii) $u \rightarrow g(x, u)$ dérivable et telle que :

$$
\forall M, \sup _{\substack{|u| \leq M \\ x \in \mathbb{R}}}\left|\frac{\partial g}{\partial u}(x, u)\right| \leq K_{M}<+\infty ;
$$

(iii) $g(x, u)=o\left(u^{p-1}\right)$ quand $u \rightarrow 0$, uniformément en $x$;

(iv) il existe $a \in L^{\infty}(\Omega)$, a $(x)>0$ dans $\Omega$, et $\left.\gamma \in\right] p, \bar{\gamma}[$ avec $\bar{\gamma}=+\infty$ si $p \geq N$ et $\bar{\gamma}=\frac{N p}{N-p}$ si $p<N$ tels que, pour u suffisamment grand :

$$
g(x, u)=a(x) u^{\gamma-1} .
$$

Alors $\mathcal{P}$ admet au moins une solution $u$.

THÉORÈME 3.-Sous les hypothèses du théorème 2, $u \in L^{\infty}(\Omega)$, ne s'annule pas dans $\Omega$ et possède les propriétés de régularité suivantes :

$$
\begin{array}{ll}
\text { Si } 1<p \leq 2, & u \in \mathcal{C}^{1, \alpha}(\Omega) \cap W_{l o c}^{2, p}(\Omega) ; \\
\text { si } p>2, & u \in \mathcal{C}^{1, \alpha}(\Omega) \cap B_{\infty, \text { loc }}^{p^{*}, p}(\Omega) .
\end{array}
$$

Démonstration du théorème 2.- Il suffit d'adapter au problème $(\mathcal{P})$ la démonstration de Nirenberg [9] en appliquant le lemme de Passe-Montagne à la fonction $J(u)=A(u)-B(u)$ où

$$
\begin{aligned}
A(u) & =\frac{1}{p} \int_{\Omega}|\nabla u|^{p}=\frac{1}{p}\|u\|_{X}^{p}, & X & =W_{0}^{1, p}(\Omega) \\
B(u) & =\int_{\Omega} G(x, u(x)) d x, & G(x, u) & =\int_{0}^{u} g(x, v) d v .
\end{aligned}
$$




\section{F. de Thélin}

a) Pour montrer que $A$ est de classe $C^{1}$ sur $X$, il suffit de voir que d'après une relation algébrique de SIMON [14] et l'inégalité de HöldER :

$$
\begin{aligned}
\left\|A^{\prime}\left(u_{1}\right)-A^{\prime}\left(u_{2}\right)\right\|_{X^{\prime}} & \leq\left\|F\left(\nabla u_{1}\right)-F\left(\nabla u_{2}\right)\right\|_{p^{*}} \\
& \leq c\left\|\nabla u_{1}-\nabla u_{2}\right\|_{p}^{\alpha-1}\left\{\left\|\nabla u_{1}\right\|_{p}+\left\|\nabla u_{2}\right\|_{p}\right\}^{p-\alpha}
\end{aligned}
$$

avec $\alpha=p$ si $1<p \leq 2, \alpha=2$ si $p>2$.

De plus $g$ étant régulière, $B$ est de classe $\mathcal{C}^{\mathbf{1}}$.

b) Soit $\left(u_{n}\right) \subset X$ telle que $J\left(u_{n}\right)$ soit bornée et $J^{\prime}\left(u_{n}\right) \rightarrow 0$. On montre comme dans [9] que $\left\|u_{n}\right\|_{X}$ est bornée; par compacité de l'injection de $X$ dans $L^{\gamma}(\Omega)$, une sous-suite encore notée $u_{n}$, converge dans $L^{\gamma}(\Omega)$. Soit

$$
\begin{aligned}
I_{n, m} & =\int_{\Omega}\left[F\left(\nabla u_{n}\right)-F\left(\nabla u_{m}\right)\right] \cdot\left(\nabla u_{n}-\nabla u_{m}\right) \\
I_{n, m} & =\left\langle J^{\prime}\left(u_{n}\right)-J^{\prime}\left(u_{m}\right), u_{n}-u_{m}\right\rangle+\int_{\Omega}\left[g\left(x, u_{n}\right)-g\left(x, u_{m}\right)\right]\left(u_{n}-u_{m}\right) .
\end{aligned}
$$

Il est facile de voir que $\lim _{n, m \rightarrow+\infty} I_{n, m}=0$.

Or $F$ vérifie la relation algébrique [14] :

$$
\begin{aligned}
\left|\nabla u_{n}-\nabla u_{m}\right|^{p} \leq c\left\{\left[F\left(\nabla u_{n}\right)-F\left(\nabla u_{m}\right)\right] \cdot\right. & \left.\left(\nabla u_{n}-\nabla u_{m}\right)\right\}^{\alpha / 2} \\
& \left(\left|\nabla u_{n}\right|^{p}+\left|\nabla u_{m}\right|^{p}\right)^{1-\alpha / 2}
\end{aligned}
$$

avec $\alpha=p$ si $1<p \leq 2$ et $\alpha=2$ si $p>2$.

Par application de l'inégalité de Hölder, on en déduit :

$$
\left\|u_{n}-u_{m}\right\|_{X}^{p} \leq c\left(I_{n, m}\right)^{\alpha / 2}\left\{\left\|u_{n}\right\|_{X}^{p}+\left\|u_{m}\right\|_{X}^{p}\right\}^{1-\alpha / 2}
$$

et donc $\lim _{n, m}\left\|u_{n}-u_{m}\right\|_{X}=0$.

$X$ étant complet, la suite $u_{n}$ converge dans $X$ et la condition de Palais-Smale est vérifiée.

c) $J(0)=0$ et, étant donné $u_{0}$ tel que $J\left(u_{0}\right)>0, \lim _{\mu \rightarrow+\infty} J\left(\mu u_{0}\right)=$ $-\infty$, d'où l'existence de $v_{0} \neq 0$ tel que $J\left(v_{0}\right)=0$.

Enfin pour tout $\varepsilon>0$, on montre comme [9] que

$$
J(u) \geq \frac{1}{p}\|u\|_{X}^{p}-C\left(\varepsilon\|u\|_{X}^{p}+C_{\varepsilon}\|u\|_{X}^{\gamma}\right)
$$

et donc pour $\|u\|_{X}=\alpha$ assez petit, $J(u) \geq \beta>0$. 
Résultats d'existence et de non-existence pour une e.d.p. elliptique non linéaire

De a), b), c), on déduit l'existence de $u \not \equiv 0, u \in W_{0}^{1, p}(\Omega)$, tel que :

$$
\forall v \in X, \quad \int_{\Omega} F(\nabla u) \cdot \nabla v-\int_{\Omega} g(x, u) v=0
$$

$g(x, u)$ pouvant être prise $\geq 0$ pour tout $u$, on montre en prenant $v=\bar{u}$ que $u \geq 0$.

Démonstration du théorème 3.- Montrons d'abord que $u \in L^{\infty}(\Omega)$. La démonstration est quasiment celle d'OTANI [12] que nous allons inclure ici pour que l'exposé soit plus complet. Le résultat étant évident si $p>N$, nous supposons $p \leq N$ et définissons :

$$
\pi= \begin{cases}\frac{N p}{N-p} & \text { si } p<N \\ \max (2 p, 2 \gamma) & \text { si } p=N\end{cases}
$$

Pour $k \in \mathbf{N}$, on définit la suite :

$$
q_{k}=\left\{\left(\frac{\pi}{p}\right)^{k-1}(\pi-\gamma)-(p-\gamma)\right\} \frac{\pi}{\pi-p}
$$

$q_{1}=\pi$ et pour tout $k$, on a :

$$
q_{k+1}=\left(q_{k}+p-\gamma\right) \frac{\pi}{p}
$$

Lemme 3. $-\forall k \in \mathbf{N}^{*}, u \in L^{q_{k}}(\Omega)$ et de plus $\|u\|_{\infty}=\widetilde{\lim }\|u\|_{q_{k}}<+\infty$.

Démonstration du lemme 3.- Montrons par récurrence que $u \in L^{q_{k}}(\Omega)$.

La propriété est vraie pour $k=1$, d'après le théorème 2 , l'injection de $W^{1, p}$ dans $L^{\pi}$ étant continue [1].

Supposons donc $u \in L^{q_{k}}$. Les hypthèses du théorème 2 donnent la majoration :

$$
\begin{gathered}
|g(x, u)| \leq \mu+\lambda|u|^{\gamma-1} \\
\|g(\cdot, u)\|_{q_{k} /(\gamma-1)} \leq \mu(\operatorname{mes} \Omega)^{(\gamma-1) / q_{k}}+\lambda\|u\|_{q_{k}}^{\gamma-1} .
\end{gathered}
$$

Il existe alors une suite $\left(v_{\varepsilon}\right) \subset \mathcal{D}(\Omega)$ telle que :

$$
\lim _{\varepsilon \rightarrow 0}\left\|v_{\varepsilon}-g(\cdot, u)\right\|_{q_{k} /(\gamma-1)}=0 .
$$


F. de Thélin

Considérons $u_{\varepsilon}$ la solution classique, $u_{\varepsilon} \in \mathcal{C}^{2}(\bar{\Omega})$ du problème

$$
\left(\mathcal{P}_{\varepsilon}\right) \begin{cases}-\Delta_{p}^{\varepsilon} u_{\varepsilon}=v_{\varepsilon} & \text { dans } \Omega \\ u_{\varepsilon}=0 & \text { sur } \partial \Omega\end{cases}
$$

où $\Delta_{p}^{\varepsilon} u_{\varepsilon}=\operatorname{div}\left[\left(\left|\nabla u_{\varepsilon}\right|^{2}+\varepsilon\right)^{(p-2) / 2} \nabla u_{\varepsilon}\right]$.

a) Montrons que $u_{\varepsilon}$ converge faiblement vers $u$ dans $W_{0}^{1, p}(\Omega)$. Notant $\Omega_{\varepsilon}=\left\{x \in \Omega:\left|\nabla u_{\varepsilon}\right|^{2} \geq \varepsilon\right\}$ et $\alpha=\max \left(1,2^{(2-p) / 2}\right)$, on a :

$$
\int_{\Omega_{\varepsilon}}\left|\nabla u_{\varepsilon}\right|^{p} \leq \alpha \int_{\Omega}\left(\left|\nabla u_{\varepsilon}\right|^{2}+\varepsilon\right)^{(p-2) / 2}\left|\nabla u_{\varepsilon}\right|^{2}=\varepsilon \int_{\Omega} v_{\varepsilon} u_{\varepsilon} .
$$

D'où avec l'inégalité de Hōlder :

$$
\int_{\Omega}\left|\nabla u_{\varepsilon}\right|^{p} \leq \alpha\left\|v_{\varepsilon}\right\|_{\gamma^{*}}\left\|u_{\varepsilon}\right\|_{\gamma}+\varepsilon^{p / 2} \operatorname{mes}(\Omega) .
$$

La suite $v_{\varepsilon}$ est bornée dans $L^{\gamma^{*}}$ puisque $\gamma^{*}=\frac{\gamma}{\gamma-1}<\frac{q_{k}}{\gamma-1} ; W^{1, p}$ s'injecte continuement dans $L^{\gamma}$; il résulte alors de (3) :

$$
\left\|\nabla u_{\varepsilon}\right\|_{p}^{p} \leq C\left\|\nabla u_{\varepsilon}\right\|_{p}+\varepsilon^{p / 2} \operatorname{mes}(\Omega) .
$$

Donc la suite $u_{\varepsilon}$, bornée dans $W_{0}^{1}, p(\Omega)$, admet une sous-suite, encore notée $u_{\varepsilon}$, faiblement convergente vers $w \in W_{0}^{1, p}(\Omega)$. Définissons dans $L^{p}(\Omega)$ :

$$
\Phi_{\varepsilon}(z)= \begin{cases}\int_{\Omega}\left(|\nabla z|^{2}+\varepsilon\right)^{p / 2} & \text { si } z \in W_{0}^{1, p}(\Omega) \\ +\infty & \text { sinon. }\end{cases}
$$

$-\Delta_{p}^{\varepsilon}$ étant le sous-différentiel de $\Phi_{\varepsilon}$, on a :

$$
\forall z \in L^{p}(\Omega), \quad \Phi_{\varepsilon}(z) \geq \Phi_{\varepsilon}\left(u_{\varepsilon}\right)+\int_{\Omega} v_{\varepsilon}\left(z-u_{\varepsilon}\right) .
$$

Sachant que, pour tout $z, \Phi_{\varepsilon}(z) \geq \Phi_{0}(z)$, il vient, par semi-continuité inférieure faible de $\Phi_{0}$ dans $W^{1, p}$ :

$$
\forall z \in W_{0}^{1, p}(\Omega), \quad \Phi_{0}(z) \geq \Phi_{0}(w)+\int_{\Omega} g(\cdot, u)(z-u)
$$

qui implique $-\Delta_{p} w=g(\cdot, u)$ et donc $w=u$ par unicité. 
Résultats d'existence et de non-existence pour une e.d.p. elliptique non linéaire

b) Montrons que $u \in L^{q_{k+1}}(\Omega)$. Soit $m \in \mathbf{N}, 1 \leq m \leq k$. Multipliant l'équation $\operatorname{par}\left|u_{\varepsilon}\right|^{q_{m}-\gamma} u_{\varepsilon}$, on a comme dans (3) :

$$
\begin{gathered}
\left(q_{m}-\gamma+1\right) \int_{\Omega}\left|\nabla u_{\varepsilon}\right|^{p}\left|u_{\varepsilon}\right|^{q_{m}-\gamma} \leq \alpha \int_{\Omega} v_{\varepsilon}\left|u_{\varepsilon}\right|^{q_{m}-\gamma} u_{\varepsilon}+\varepsilon^{p / 2} \int_{\Omega}\left|u_{\varepsilon}\right|^{q_{m}-\gamma} \\
\left.\leq \alpha\left\|v_{\varepsilon}\right\|_{q_{m} /(\gamma-1)}\left\|\left|u_{\varepsilon}\right|^{q_{m}-\gamma+1}\right\|_{\left(q_{m} /(\gamma-1)\right.}\right)^{*} \\
\quad+\varepsilon^{p / 2}(\operatorname{mes}(\Omega))^{\gamma / q_{m}}\left\|\left|u_{\varepsilon}\right|^{q_{m}-\gamma}\right\|_{q_{m} /\left(q_{m}-\gamma\right)} \\
\leq\left(C_{1}+C_{2}\left\|u_{\varepsilon}\right\|_{q_{m}}^{\gamma-1}\right)\left\|u_{\varepsilon}\right\|_{q_{m}}^{q_{m}-\gamma+1}+C_{3}\left\|u_{\varepsilon}\right\|_{q_{m}}^{q_{m}-\gamma} .
\end{gathered}
$$

D'où, par application de l'inégalité de Young :

$$
\left(q_{m}-\gamma+1\right) \int_{\Omega}\left|\nabla u_{\varepsilon}\right|^{p}\left|u_{\varepsilon}\right|^{q_{m}-\gamma} \leq C_{4}+C_{5}\left\|u_{\varepsilon}\right\|_{q_{m}}^{q_{m}} .
$$

Par ailleurs il existe une constante $K$ telle que [1] :

$$
\forall w \in W_{0}^{1, p}(\Omega), \quad\|w\|_{\pi} \leq K\|\nabla w\|_{p},
$$

donc :

$$
\begin{aligned}
\left\|u_{\varepsilon}\right\|_{q_{m+1}}^{q_{m}+p-\gamma}=\left\|\left|u_{\varepsilon}\right|^{1+\frac{q_{m}-\gamma}{p}}\right\|_{\pi}^{p} & \leq K^{p} \int_{\Omega}\left|\nabla\left(u_{\varepsilon}^{1+\frac{q_{m}-\gamma}{p}}\right)\right|^{p} \\
& \leq\left(1+\frac{q_{m}-\gamma}{p}\right)^{p} K^{p} \int_{\Omega}\left|\nabla u_{\varepsilon}\right|^{p}\left|u_{\varepsilon}\right|^{q_{m}-\gamma} .
\end{aligned}
$$

Il en résulte alors avec (4):

$$
\left\|u_{\varepsilon}\right\|_{q_{m+1}}^{q_{m}+p-\gamma} \leq\left\{C_{6}+C_{7}\left\|u_{\varepsilon}\right\|_{q_{m}}^{q_{m}}\right\}\left(q_{m}+p-\gamma\right)^{p} .
$$

On en déduit par récurrence sur $m$ que $u_{\varepsilon}$ est borné dans chaque $L^{q_{m}}$, $1 \leq m \leq k+1$ et donc $u \in L^{q_{k+1}}(\Omega)$.

c) La relation ci-dessous montre en particulier que $u_{\varepsilon}$ est borné dans $L^{q_{k+1}}$; sachant de plus que $\nabla u_{\varepsilon}$ est borné dans $L^{p}$, il résulte du théorème de RELLICH (voir par exemple [15], p. 22) qu'une sous suite extraite de $u_{\varepsilon}$ converge fortement vers $u$ dans $L^{q_{k}}$. On obtient donc à la limite la relation :

$$
\left(\|u\|_{q_{k+1}}^{q_{k+1}}\right)^{\frac{p}{\pi}} \leq\left\{C_{6}+C_{7}\|u\|_{q_{k}}^{q_{k}}\right\}\left(q_{k}+p-\gamma\right)^{p} .
$$

Remarquant que $q_{k} \leq \pi\left(\frac{\pi}{p}\right)^{k-1}$ et posant :

$$
\begin{aligned}
& a=\frac{\pi}{p}, \quad b=\frac{\pi}{p} \log \max \left\{1, C_{6}+C_{7}\right\}, \\
& E_{k}=q_{k} \log \max \left\{1,\|u\|_{q_{k}}\right\}, \quad r_{k}=b+\pi(k-1) \log a,
\end{aligned}
$$


il vient :

$$
E_{k+1} \leq r_{k}+a E_{k} \leq r_{k}+a r_{k-1}+\cdots+a^{k-1} r_{1}+a^{k} E_{1} .
$$

Des calculs élémentaires donnent alors :

$$
E_{k+1} \leq a^{k}\left\{E_{1}+\frac{b}{a-1}+\frac{\pi \log a}{(a-1)^{2}}\right\}=d a^{k} .
$$

D'où l'on tire

$$
\|u\|_{\infty} \leq \varlimsup_{k \rightarrow+\infty} \exp \left(\frac{E_{k}}{q_{k}}\right) \leq \varlimsup_{k \rightarrow+\infty} \exp \left(\frac{d a^{k-1}}{q_{k}}\right) \leq \exp \left(\frac{d}{\pi}\right) . \square
$$

Fin de la Démonstration du ThÉorème 3 .- Grâce au lemme 3, la fin de la démonstration est analogue à celle du théorème 1 .

\section{Non existence}

Nous dirons que l'ouvert $\Omega$ est étoilé [respectivement strictement étoilé] si, pour un choix convenable de l'origine, $n(x) \cdot x \geq 0$ [respectivement $n(x) \cdot x \geq \rho>0]$ pour tout $x \in \partial \Omega$, où $n(x)$ désigne la normale extérieure en $x \in \partial \Omega$.

Dans ce paragraphe, nous nous limiterons au cas où la fonction $g$ ne dépend que de $u$; le résultat principal est le théorème suivant :

THÉORÈME 4. - Soit $g$ une fonction continue sur $\mathbf{R}$ telle que :

$$
g(u)>0 \text { pour } u>0 .
$$

Considérons :

$$
G(u)=\int_{0}^{|u|} g(v) d v
$$

On suppose que l'une des deux hypothèses suivantes est réalisée :

(i) $\Omega$ est étoilé et $u \rightarrow \frac{G(u)}{u^{N p /(N-p)}}$ est strictement croissante;

(ii) $\Omega$ est strictement étoilé et $u \rightarrow \frac{G(u)}{u^{N p /(N-p)}}$ est croissante.

Alors le problème $(\mathcal{P})$ n'admet pas de solution $u \in L^{\infty}(\Omega)$.

Remarque.- Ni et SERRIN [8] ont prouvé un résultat analogue pour des solutions radiales singulières. 
Résultats d'existence et de non-existence pour une e.d.p. elliptique non linéaire

Comme dans [8], [17], la démonstration de ce théorème utilise essentiellement une égalité de type Pohozaev [13]. Nous allons reprendre en partie l'idée de la démonstration d'OTANi [12].

Démonstration. - Par l'absurde. Supposons que $(\mathcal{P})$ admette une solution $u \in L^{\infty}(\Omega)$; alors $g(u) \in L^{\infty}(\Omega)$ et il existe $\left(v_{\varepsilon}\right) \subset \mathcal{D}(\Omega)$ telle que :

$$
\lim _{\varepsilon \rightarrow 0}\left\|v_{\varepsilon}-g(u)\right\|_{p^{*}}=0
$$

Soit $u_{\varepsilon}$ la solution classique $u \in \mathcal{C}^{2}(\bar{\Omega})$ du problème $(\mathcal{P})$. On note $F_{i}^{\varepsilon}=$ $\left(\left|\nabla u_{\varepsilon}\right|+\varepsilon\right)^{p-2 / 2} D_{i} u_{\varepsilon}$ et $F^{\varepsilon}=\left(F_{1}^{\varepsilon}, \ldots, F_{N}^{\varepsilon}\right)$.

LEMME 4. - $u_{\varepsilon}$ converge faiblement vers $u$ dans $W_{0}^{1, p}(\Omega)$.

Démonstration du lemme 4.- Reprenons la démonstration du lemme 3; nous avons d'après $(2)$ :

$$
\int_{\Omega}\left|\nabla u_{\varepsilon}\right|^{p} \leq \alpha \int_{\Omega} v_{\varepsilon} u_{\varepsilon}+\varepsilon^{p / 2} \operatorname{mes}(\Omega) .
$$

D'après l'inégalité de PoIncaré il existe $c>0$ tel que :

$$
\left\|u_{\varepsilon}\right\|_{p}^{p} \leq c\left\|\nabla u_{\varepsilon}\right\|_{p}^{p}
$$

d'où

$$
\left\|\nabla u_{\varepsilon}\right\|_{p}^{p} \leq \frac{1}{2}\left\|\nabla u_{\varepsilon}\right\|_{p}^{p}+\left(\frac{2 c}{p}\right)^{p^{*} / p} \frac{\alpha^{p^{*}}}{p^{*}}\left\|v_{\varepsilon}\right\|_{p^{*}}^{p^{*}}+\varepsilon^{p / 2} \operatorname{mes}(\Omega) .
$$

On en déduit que $u_{\varepsilon}$ est borné dans $W_{0}^{1, p}(\Omega)$ et, comme pour le lemme 3, converge faiblement vers $u$ dans $W_{0}^{1, p}(\Omega)$.

LEMME 5.- - vérifie la relation suivante :

$$
\begin{gathered}
-p \int_{\Omega}\left(x . \nabla u_{\varepsilon}\right) v_{\varepsilon}-(N-p) \int_{\Omega} u_{\varepsilon} v_{\varepsilon}-N \varepsilon \int_{\Omega}\left(\left|\nabla u_{\varepsilon}\right|^{2}+\varepsilon\right)^{(p-2) / 2} \\
=(p-1) \int_{\partial \Omega}\left(\left|\nabla u_{\varepsilon}\right|^{2}+\varepsilon\right)^{(p-2) / 2}\left|\nabla u_{\varepsilon}\right|^{2}(x \cdot n) d \sigma \\
-\varepsilon \int_{\partial \Omega}\left(\left|\nabla u_{\varepsilon}\right|^{2}+\varepsilon\right)^{(p-2) / 2}(x \cdot n) d \sigma .
\end{gathered}
$$


Démonstration du lemme $5 .-u_{\varepsilon}$ étant constant sur $\partial \Omega, \nabla u_{\varepsilon}$ est porté par la normale extérieure $n$ en $x \in \partial \Omega$ et il vient par la formule de GaUsS :

$$
\begin{aligned}
& p \int_{\partial \Omega}\left(\left|\nabla u_{\varepsilon}\right|^{2}+\varepsilon\right)^{(p-2) / 2}\left|\nabla u_{\varepsilon}\right|^{2}(x \cdot n) d \sigma \\
& =p \int_{\partial \Omega}\left(x \cdot \nabla u_{\varepsilon}\right)\left(F^{\varepsilon} \cdot n\right) d \sigma \\
& =p \int_{\Omega}\left(x \cdot \nabla u_{\varepsilon}\right) \sum_{i=1}^{N} \frac{\partial}{\partial x_{i}} F_{i}^{\varepsilon}+p \int_{\Omega} \sum_{i=1}^{N} \frac{\partial}{\partial x_{i}}\left(x \cdot \nabla u_{\varepsilon}\right) F_{i}^{\varepsilon} \\
& =p \int_{\Omega}\left(x \cdot \nabla u_{\varepsilon}\right) \Delta_{p}^{\varepsilon} u_{\varepsilon}+p \int_{\Omega} \sum_{i=1}^{N} \frac{\partial u_{\varepsilon}}{\partial x_{i}} F_{i}^{\varepsilon} \\
& \quad+p \int_{\Omega} \sum_{i=1}^{N} \sum_{j=1}^{N} x_{j}\left(\left|\nabla u_{\varepsilon}\right|^{2}+\varepsilon\right)^{(p-2) / 2} \frac{\partial u_{\varepsilon}}{\partial x_{i}} \frac{\partial^{2} u_{\varepsilon}}{\partial x_{i} \partial x_{j}} \\
& =-p \int_{\Omega}\left(x \cdot \nabla u_{\varepsilon}\right) v_{\varepsilon}+p \int_{\Omega}\left(\left|\nabla u_{\varepsilon}\right|^{2}+\varepsilon\right)^{(p-2) / 2}\left|\nabla u_{\varepsilon}\right|^{2} \\
& \quad+\int_{\Omega} \sum_{j=1}^{N} x_{j} \frac{\partial}{\partial x_{j}}\left(\left|\nabla u_{\varepsilon}\right|^{2}+\varepsilon\right)^{p / 2} .
\end{aligned}
$$

De même pour la formule de Gauss :

$$
\begin{aligned}
\int_{\partial \Omega}\left(\left|\nabla u_{\varepsilon}\right|^{2}+\varepsilon\right)^{\frac{p}{2}}(x \cdot n) d \sigma & \\
& =N \int_{\Omega}\left(\left|\nabla u_{\varepsilon}\right|^{2}+\varepsilon\right)^{p / 2}+\int_{\Omega} \sum_{j=1}^{N} x_{j} \frac{\partial}{\partial x_{j}}\left(\left|\nabla u_{\varepsilon}\right|^{2}+\varepsilon\right)^{p / 2} .
\end{aligned}
$$

Retranchant membre à membre ces deux relations, on déduit (5) puisque :

$$
\int_{\Omega}\left(\left|\nabla u_{\varepsilon}\right|^{2}+\varepsilon\right)^{(p-2) / 2}\left|\nabla u_{\varepsilon}\right|^{2}=-\int_{\Omega}\left(\Delta_{p}^{\varepsilon} u_{\varepsilon}\right) u_{\varepsilon}=\int_{\Omega} v_{\varepsilon} u_{e} . \square
$$

LEMME 6. - Si $\Omega$ est étoilé, on a les majorations suivantes :

$$
\begin{array}{cc}
\varepsilon \int_{\partial \Omega}\left(\left|\nabla u_{\varepsilon}\right|^{2}+\varepsilon\right)^{(p-2) / 2}(x \cdot n) d \sigma \leq \varepsilon^{p / 2} \int_{\partial \Omega}(x \cdot n) d \sigma & \text { si } 1<p \leq 2 \\
\varepsilon \int_{\partial \Omega}\left(\left|\nabla u_{\varepsilon}\right|^{2}+\varepsilon\right)^{(p-2) / 2}(x \cdot n) d \sigma \leq \frac{2 \varepsilon^{p / 2}}{p} \int_{\partial \Omega}(x \cdot n) d \sigma & \\
+\frac{p-2}{p} \int_{\partial \Omega}\left(\left|\nabla u_{\varepsilon}\right|^{2}+\varepsilon\right)^{p / 2}(x \cdot n) d \sigma & \text { si } p>2 .
\end{array}
$$


Résultats d'existence et de non-existence pour une e.d.p. elliptique non linéaire

Démonstration. - Immédiate.

Démonstration du théorème 9 , partie (i) . $-\Omega$ étant étoilé, des lemmes 5 et 6 on déduit,

$$
\begin{aligned}
& \text { pour } 1<p \leq 2: \\
& -p \int_{\Omega}\left(x \cdot \nabla u_{\varepsilon}\right) v_{\varepsilon}-(N-p) \int_{\Omega} u_{\varepsilon} v_{\varepsilon} \\
& \geq(p-1) \int_{\partial \Omega}\left(\left|\nabla u_{\varepsilon}\right|^{2}+\varepsilon\right)^{(p-2) / 2}\left|\nabla u_{\varepsilon}\right|^{2} \cdot(x \cdot n) d \sigma-\varepsilon^{p / 2} \int_{\partial \Omega}(x \cdot n) d \sigma \\
& \geq-\varepsilon^{p / 2} \int_{\partial \Omega}(x \cdot n) d \sigma
\end{aligned}
$$

et pour $p>2$ :

$$
\begin{aligned}
& -p \int_{\Omega}\left(x \cdot \nabla u_{\varepsilon}\right) v_{\varepsilon}-(N-p) \int_{\Omega} u_{\varepsilon} v_{\varepsilon} \\
& \geq[(p-1)-(p-2) / p] \int_{\partial \Omega}\left(\left|\nabla u_{\varepsilon}\right|^{2}+\varepsilon\right)^{p / 2}(x \cdot n) d \sigma-\frac{2 \varepsilon^{p / 2}}{p} \int_{\partial \Omega}(x \cdot n) d \sigma \\
& \geq-\frac{2 \varepsilon^{p / 2}}{p} \int_{\partial \Omega}(x \cdot n) d \sigma .
\end{aligned}
$$

Quand $\varepsilon \rightarrow 0$, il en résulte avec le lemme 4 , pour $1<p<+\infty$ :

$$
-p \int_{\Omega}(x \cdot \nabla u) g(u)-(N-p) \int_{\Omega} u g(u) \geq 0 .
$$

Or $G(u)$ appartient à $W_{0}^{1, p}(\Omega)$ et donc :

$$
\int_{\Omega}(x \cdot \nabla u) g(u)=\int_{\Omega} \sum_{i=1}^{N} x_{i} \frac{\partial}{\partial x_{i}} G(u)=-N \int_{\Omega} G(u) .
$$

Enfin, sachant que $u \rightarrow \frac{G(u)}{u^{N p /(N-p)}}$ est strictement croissante.

$$
-u^{N p /(N-p)} g(u)+\frac{N p}{(N-p)} u^{-1+N p /(N-p)} G(u)<0
$$

d'où une contradiction avec $(6),(7),(8)$.

Démonstration du théorème 3 , partie (ii) . - Supposons $\Omega$ strictement étoilé. Les lemmes 5 et 6 donnent toujours :

$$
\begin{aligned}
& -p \int_{\Omega}\left(x \cdot \nabla u_{\varepsilon}\right) v_{\varepsilon}-(N-p) \int_{\Omega} u_{\varepsilon} v_{\varepsilon} \\
& \quad \geq \min \left(\frac{2}{p}, p-1\right) \int_{\partial \Omega}\left(\left|\nabla u_{\varepsilon}\right|^{2}+\varepsilon\right)^{p / 2}(x \cdot n) d \sigma-\varepsilon^{p / 2} \int_{\partial \Omega}(x \cdot n) d \sigma .
\end{aligned}
$$




$$
\text { F. de Thélin }
$$

Or d'après HöLDER :

$$
\begin{aligned}
\int_{\partial \Omega} & \left(\left|\nabla u_{\varepsilon}\right|^{2}+\varepsilon\right)^{p / 2}(x \cdot n) d \sigma \\
& \geq \frac{\rho}{(\operatorname{mes} \partial \Omega)^{1 /(p-1)}}\left|\int_{\partial \Omega}\left(\left|\nabla u_{\varepsilon}\right|^{2}+\varepsilon\right)^{(p-1) / 2} d \sigma\right|^{p /(p-1)} \\
& \geq \frac{\rho}{(\operatorname{mes} \partial \Omega)^{1 /(p-1)}}\left|\int_{\partial \Omega} F^{\varepsilon} \cdot n d \sigma\right|^{p^{*}} \\
& \geq \frac{\rho}{(\operatorname{mes} \partial \Omega)^{1 /(p-1)}}\left|\int_{\Omega} \Delta_{p}^{\varepsilon} u_{\varepsilon}\right|^{p^{*}} \\
= & \frac{\rho}{(\operatorname{mes} \partial \Omega)^{1 /(p-1)}}\left|\int_{\Omega} v_{\varepsilon}\right|^{p^{*}} .
\end{aligned}
$$

Quand $\varepsilon \rightarrow 0$, on en déduit avec (9) et le lemme 4 :

$$
N p \int_{\Omega} G(u)-(N-p) \int_{\Omega} u g(u) \geq \frac{\rho \min \left(\frac{2}{p}, p-1\right)}{(\operatorname{mes} \partial \Omega)^{1 /(p-1)}}\left|\int_{\Omega} g(u)\right|^{p^{*}}>0
$$

d'où une contradiction. $\square$

Remerciements. - L'auteur remercie M. OTANI pour ses intéressantes remarques.

\section{Références}

[1] Adams (R.A) .- Sobolev Spaces, Academic Press, 1975.

[2] Deuel-Hess .-A criterion for the existence of solutions of nonlinear elliptic boundary value problems, Proc. Royal Soc. Edimburgh A, vol. 74, 1975, p. 4954.

[3] Diaz (J.I.) . - Nonlinear partial differential equations and free boundaries, I-Elliptic equations, Pitmann n ${ }^{\circ}$ 106, 1985.

[4] Diaz-SaA .- Uniqueness of non-negative solutions for elliptic nonlinear diffusion equations with a general perturbation term, Proc. VII - Cedya, Santander, 1985.

[5] Lions (J.L.).-Quelques méthodes de résolution des problèmes aux limites non linéaires, Dunod, 1969.

[6] NI-SERRIN .- - Nonexistence theorems for quasilinear partial differential equations, Rendiconti Circolo Mat. Palermo, Suppl. Studies.

[7] Ni-SERRIN .- Existence and nonexistence theorems for ground states for quasilinear partial differential equations, Proc. Acad. Lincei (à paraître). 
Résultats d'existence et de non-existence pour une e.d.p. elliptique non linéaire

[8] NI-SERRIN .- Nonexistence theorems for singular solutions of quasilinear partial differential equations, Comm. Pure Appl Math, 34, 1986, p. 379-399.

[9] Nirenberg .- Variational and topological method in nonlinear problems, Bulletin of the A.M.S., vol. $4 \mathrm{n}^{\circ}$ 3, 1981, p. 267-302.

[10] OTANI (M.) .- On certain second order ordinary differential equations associated with Sobolev - Poincaré - type inequalities, Nonlinear Analysis T.M.A. $n^{\circ} 8,1984$, p. 1255-1270.

[11] Otani (M.) .- A remark on certain nonlinear elliptic equations, Proc. Fac. Sci. Tokai Univ. $n^{\circ} 19,1984$, p. 23-28.

[12] OTANI (M.) . - Existence and nonexistence of nontrivial solutions of some nonlinear degenerate elliptic equations, J. Functional Analysis, vol. $76 n^{\circ} 1,1988$, p. 140-159.

[13] Pohozaev (S.I.) - - Eigenfunctions of the equation $\Delta u+\lambda f(u)=0$, Soviet. Math. Dokl. $n^{\circ} 6,1965$, p. 1408-1411.

[14] Simon (J.) .- Sur des équations aux dérivées partielles non-linéaires, Thèse, Paris, 1977.

[15] Strauss (W.A.). - The energy method in non linear partial differential equations, Notas de Matematica $n^{\circ} 47$, Rio de Janeiro, 1969.

[16] De Thelin (F.) .- Local regularity properties for the solutions of a nonlinear partial differential equation, Nonlinear Analysis T.M.A. vol. $6 \mathrm{n}^{\circ} 8,1982$, p. 839844.

[17] De Thelin (F.) .- Quelques résultats d'existence et de non existence pour une E.D.P. elliptique non linéaire, C.R. Acad. Sc. Paris, t. 299, série I $n^{\circ} 18,1984$, p. 911-914.

[18] De Thelin (F.) .- Sur l'espace propre associé à la première valeur propre du pseudo-laplacien, C.R. Acad. Sc. Paris, t. 303, série I, $n^{\circ} 8,1986$, p. 355-357.

[19] TolksDorf (P.) . - On the Dirichlet problem for quasilinear equations in domains with conical boundary points, Comm. P.D.E., $n^{\circ} 8,1983$, p. 773-817.

[20] VAzQuez (J.L.) .- A strong maximum principle for somme quasilinear elliptic equations, Appl. Math. and Optimization $n^{\circ} 12,1984$, p. 191-202.

(Manuscrit reçu le 12 décembre 1986) 